\title{
Target Segmentation in Scenes with Diverse Background
}

\author{
Christina Grönwall and Gustav Tolt \\ Division of Information Systems, FOI (Swedish Defence Research Agency), \\ P.O. Box 1165, SE-58111 Linköping, Sweden \\ \{christina.gronwall, gustav.tolt\}@foi.se \\ http://www.foi.se
}

\begin{abstract}
We propose a target segmentation approach based on sensor data fusion that can deal with the problem of a diverse background. Features from sensor images, including data from a laser scanner and passive sensors (cameras), are analyzed using Gaussian mixture estimation. The approach tackles some of the difficulties with Gaussian mixtures, e.g., selecting the number of initial components and a good description of data in terms of the number of Gaussian components, and determining the relevant features for the current data set. The feature selection quality is analyzed on-line. We propose a criterion that determines the quality of the resulting clusters in terms of their respective spatial distribution. The output from the analysis is used for object-background segmentation. Segmentation examples of surface-laid mines in outdoor scenes are shown.
\end{abstract}

Keywords: Feature selection, segmentation, Gaussian mixture, mine detection, cluster selection.

\section{Introduction}

Detection of small targets in complex and changing environments is a challenging problem due to factors like varying lighting conditions, shadowing effects, different physical properties of the targets, varying aspect angles of the sensors, occlusion, etc. The complex and changing background in which the targets are placed can often be described by a mixture model, but the model parameters have to be estimated from data to give a meaningful representation. Without a priori information of the most informative sensor data in a particular case, measurements of several physical phenomena are desired. As a consequence, we need a signal processing framework that can extract information from multi-faceted data and has the flexibility to handle new terrain types and a diversity of target signatures. The application in mind is detection of surface-laid land-mines in vegetation areas. The scene in Fig. 1h contains several mines of different models, and although no mines are buried they are not that easy to find.

In this paper a segmentation method based on Gaussian mixture models is proposed. It addresses some of the difficulties with Gaussian mixtures; selecting

A. Heyden and F. Kahl (Eds.): SCIA 2011, LNCS 6688, pp. 708-718, 2011.

(C) Springer-Verlag Berlin Heidelberg 2011 
the number of initial components and a good description of the data set and determining relevant features for the current data set. Assuming that data are samples from mixtures of Gaussian distributions reduces the problem partly to a "missing parameter problem". Several features are computed and evaluated to determine what combination gives the best results, using measures in the feature domain as well as estimates of the physical size of the segments. The only exploited a priori knowledge is the approximate target dimensions. Due to the complexity of the background, we propose on-line selection of both the number of components and features. We add one feature at a time and need dynamic ranking of the features and the possibility to vary the order that features are added.

Mixture models constitute a widely used approach for unsupervised learning problems. Selecting the number of components is discussed in 510 11, where [5] proposes the Minimum Message Length (MML) for component number selection and 1011] combine component and feature selection in a Bayesian framework. Bali [8] proposes a joint solution for the number of features and selection of number of components problem, taking into account both the spatial and spectral structures in data. Fauvel et al. [13] tackles the problem by fusing morphological information (spatial data properties) and the original hyperspectral data using support vector machines. Feature selection is also discussed in [412], where 12. uses the Principal Component Analysis (PCA) for feature selection. The drawbacks of PCA and other measures with unclear physical interpretation are discussed in [8]. Jimenez 9] describes a preprocessing step for reducing the number of features. Two criteria for feature selection are discussed in [4]: the scatter separability (SS) criteria and the Maximum Likelihood (ML).

Our work is inspired by [4] and [5], but we use histogram peak detection to guide the initial number of components instead of starting with several components and then reducing them. We use the MML criterion [5] to select the proper number of components. We incorporate feature selection in the component estimation and analyze the quality of the current feature selection on-line. We also propose an complement to the normalized SS measure that is based on analyzing the spatial distribution of the resulting clusters. High-dimensional mixture modeling is common for segmentation of hyper spectral images 8913. We fuse features originating from image data of various resolution (laser scanner and passive sensors). Sensor data are co-registered to pixel-correspondence. Earlier work of this approach has been reported in [7,6]. Our segmentation approach is described in Section 2. Examples on real data and analysis results are shown in Section 3. The work is discussed and concluded in Section 4.

\section{Clustering of Object and Background Data}

The main problems associated with the proposed clustering method are to a) determine the best clustering given a certain set of features, b) determine whether the addition of another feature improves the result and c) specify the order in which the features should be added. Below, we describe the basic building blocks of the method and how we address the problems. 


\subsection{Gaussian Mixture Model}

We start by recalling that the probability density function (pdf) for a Gaussian mixture can be written as

$$
\begin{aligned}
P(\mathbf{y} \mid \Theta) & =\sum_{m=1}^{k} \alpha_{m} p\left(\mathbf{y} \mid \theta_{m}\right) \\
\theta_{m} & \equiv\left[\mu_{m}, \sigma_{m}\right] \\
\Theta & \equiv\left[\theta_{1}, \ldots, \theta_{k}, \alpha_{1}, \ldots, \alpha_{k}\right] \\
p\left(\mathbf{y} \mid \theta_{m}\right) & \in N\left(\mu_{m}, \sigma_{m}^{2}\right) \\
\alpha_{m} & \geq 0, \quad m=1, \ldots, k, \quad \sum_{m=1}^{k} \alpha_{m}=1,
\end{aligned}
$$

where $\mathbf{y}=\left[y_{1}, \ldots, y_{n}\right]^{T}$ is the given feature vector with $n$ samples, $k$ is the number of Gaussian components, $p(\cdot \mid \cdot)$ is the Gaussian probability function, $\alpha_{m}$ is the relative weight between each Gaussian, $\theta_{m}$ contains the mean, $\mu_{m}$, and standard deviation, $\sigma_{m}$, for each component $m$.

\subsection{The Minimum Message Length Criterion}

When estimating the parameters with the EM algorithm it is required that the number of components is known, which is not the case for unsupervised methods. For this purpose, we use the Minimum Message Length (MML) criterion and choose the optimal number of components as the one that minimizes the MML. The underlying idea of the MML criterion is that if a short code can be built for the data, the data generation model is good 35. In the unsupervised case the MML criterion is formulated as 5 ]

$$
\begin{aligned}
L(\Theta, Y)= & \frac{D}{2} \sum_{m: \alpha_{m}>0} \log \left(\frac{n \alpha_{m}}{12}\right)+ \\
& \frac{k}{2} \log \frac{n}{12}+\frac{k(D+1)}{2}-\log p(Y \mid \Theta),
\end{aligned}
$$

where $D$ is the number of parameters specifying each component, $k$ the total number of components and $n$ the number of samples/observations.

\subsection{The EM-MML Algorithm and Initialization}

The MML criterion is added to the EM algorithm and we further on refer to the EM-MML algorithm. In order to preserve small clusters, a local maximum-based initialization was chosen over other techniques, such as random initialization or initialization based on principal directions. In order to avoid finding local maxima in a high-dimension feature space, we start with a $1 \mathrm{D}$ clustering problem and then add features sequentially until the best segmentation is obtained. The feature sorting procedure is described in Sec. 2.6.

To initialize the 1D clustering, a smoothed 1D histogram of a feature vector $Y$ is created and the location of the top $k$ local maxima in the histogram are 
chosen as start values for $\mu_{m}$. The weights $\alpha_{m}$ are distributed equally between the start components and the standard deviations are given a small constant value.

Starting from an initialization with $k$ Gaussian components, an EM estimation is made for each $m=k, \ldots, 1$. A threshold $\tau$ is set that removes model components whose weights ( $\alpha$ 's) are too small between each estimation, to avoid the problem with a singular covariance matrix. If none of the weights are close to zero, the two components with the shortest Euclidean distance are selected and of those two, the component with the smallest weight is removed. After one component has been removed, the clustering continues with the remaining ones. The clustering result giving the lowest MML value is then selected as the optimal one.

The parameters of a current $d$-dimensional model are used to initialize a higher dimension $(d+1)$ model. This is done by extending the $d$-dimensional model with the parameters from the 1-D analysis of the feature to be added. For example, assume that the current 1-D model contains three components and that a second feature containing four components is added. The 2-D model is then initialized with 12 components.

\subsection{The Normalized Scatter Separability Criterion}

The SS criterion [4] is a measure of how separated the clusters are and how compact each cluster is, the assumption being that the more separated the clusters, the better the segmentation. The SS criterion is defined as

$$
S S=\operatorname{trace}\left(S_{w}^{-1} S_{b}\right)
$$

where $S_{w}$ is a sum of the weighted covariances and $S_{b}$ is the sum of the weighted sample variance. A high value of SS equals a maximization of the between-class scatter matrix, $S_{b}$, and a minimization of the within-class scatter matrix, $S_{w}$.

To compare SS criteria for two set-ups with different number of features, the criterion is normalized with respect to dimension [4]:

$$
\overline{S S}\left(s_{j}, C_{j}\right)=S S\left(s_{j}, C_{j}\right) \cdot S S\left(s_{j+1}, C_{j}\right),
$$

where $s_{j}=\left[\mathbf{y}_{\mathbf{1}}, \ldots, \mathbf{y}_{\mathbf{j}}\right] \in Y$, where $j=2, \ldots, d-1, C_{j}$ is the number of components after estimation of $s_{j}$.

\subsection{The Spatial Scatter Criterion}

When judging whether the EM-MML clustering has successfully divided the data into object and background, existing application-specific knowledge about the expected targets should be used. The target size is known to lie within a certain interval. Ideally the background would be random and would then result in segments consisting of samples that are uniformly distributed across the scene, while segments that form some sort of spatially concentrated cluster can correspond to targets. Therefore, we propose a spatial scatter criterion $(S C)$, defined as the mean distance from the segment centroid to the samples in the 
segments. Let $\mathcal{I}_{m}$ represent the indices of all samples $x_{i}$ belonging to cluster $m$, such that

$$
\mathcal{I}_{m}=\left\{i: p\left(\mathbf{y}_{i} \mid \theta_{m}\right)=\arg \max _{l=1, \ldots, k} p\left(\mathbf{y}_{i} \mid \theta_{l}\right)\right\} .
$$

Then the spatial scatter measure for cluster $m, S C_{m}$, can be written as

$$
S C_{m}=\frac{1}{n_{m}} \sum_{i \in \mathcal{I}_{m}}\left\|x_{i}-\bar{x}_{m}\right\|,
$$

where $\|\cdot\|$ is the Euclidean distance in the real world coordinate system and $\bar{x}_{m}$ is the centroid coordinates of cluster $m$.

\subsection{Feature Sorting}

As the clustering result depends on the order in which the features are added, they have to be sorted wisely prior to the main segmentation. To solve this, every feature is subject to one-dimensional EM-MML clustering. The comparison between features is first done by sorting the corresponding clustering results according to ascending $S S$ measure and then selecting features giving clusters of acceptable $S C$ measures first (if any). As a result, we give priority to features that produce clusters of acceptable physical size that are also well separated in the feature space.

\section{$3 \quad$ Examples}

The proposed method is tested on real data from field trials. The analysis is based on optical sensors, which implies that the mines should be (partly) visible, i.e., surface laid mines. In Fig. 1 the features for one of targets are shown and the segmentation is shown in Fig. 2. In this case the target data is segmented into two parts (blue and green) and the background into one segment (red). Fig. 3 shows another example, where the result obtained with highest-ranked feature is improved as more features are added.

\subsection{Sensor Data and Data Registration}

Data was collected at different field trials [1/2] where images were captured in a forward-looking view. Three sensors were used: a scanning 3D-imaging laser radar, a passive IR camera collecting data in four SWIR/MWIR spectral bands $(1.5-5.2 \mu \mathrm{m})$, and a high-resolution camera operating in the visual range. The spatial resolution of the laser scanner and the visual range camera was in the order of $1 \mathrm{~cm}$ per point/pixel on the ground, while the resolution of the SWIR/MWIR sensor was about $2.5 \mathrm{~cm} /$ pixel.

To fuse data on the pixel level, sensor data was registered [2], i.e., transformed to a reference coordinate system (here, the local coordinate system of the laser scanner). Using the range information from the laser sensor, 3D points can readily be projected onto an image plane to support the image matching in 


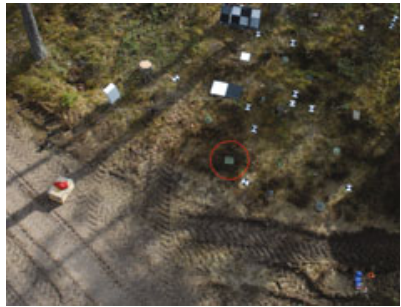

(a)

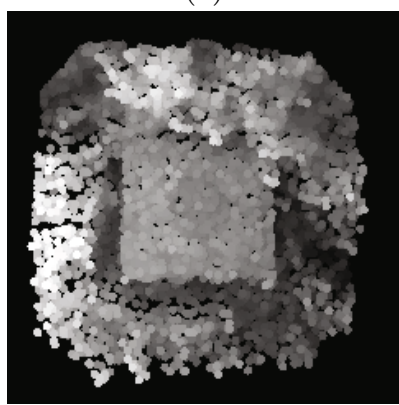

(d)

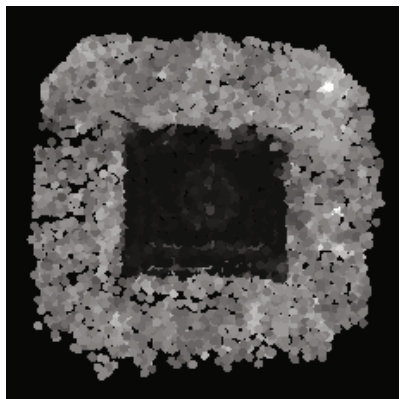

(b)

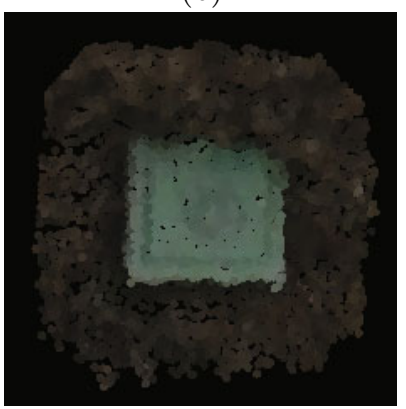

(e)

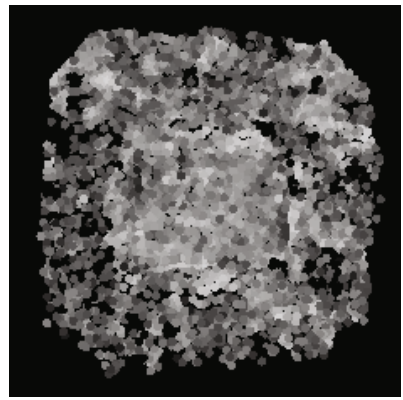

(c)

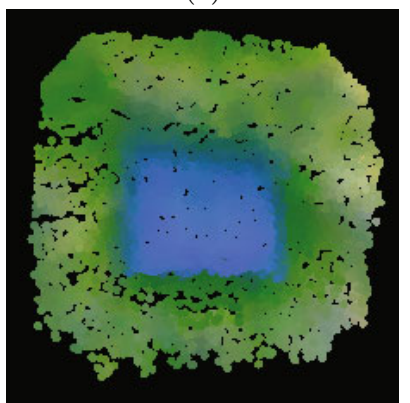

(f)

Fig. 1. (a) A forest scene, with the target under study marked with a red circle. (b) Laser intensity. (c) Surface score. (d) Height above local ground plane. (e) RGB data. (f) Three Mid-wave IR bands pseudo-colored as RGB values.

complex scenes. All objects were placed on a quite flat ground surface and an ordinary image matching approach produced results at par with the 3D based registration. A visual inspection was made to verify the overlap of the data after the transformation, although an offset of a few centimeters is possible. For the sets that contained mine data and other objects of interest ground truth target masks were created to support the evaluation of the resulting segmentation.

\subsection{Features}

Three features were generated from the laser radar images: the laser return intensity, height above the ground plane (calculated by rotation of the 3D point cloud) and a surface score. From the passive sensors (IR and visual cameras) the spectral bands (four IR bands and the color channels R, G, and B) were used as features. Each sample is associated with a $d$-dimensional feature vector and the complete data set could then be viewed as a $n \times d$ feature matrix.

The height above the ground plane and the surface score are spatial features, included to support segmentation when the spectral contrast is poor. We assume that targets consist of relatively smooth surface patches which make them different from the background. The surface score $\left(S_{x}\right)$ is used to represent the degree 

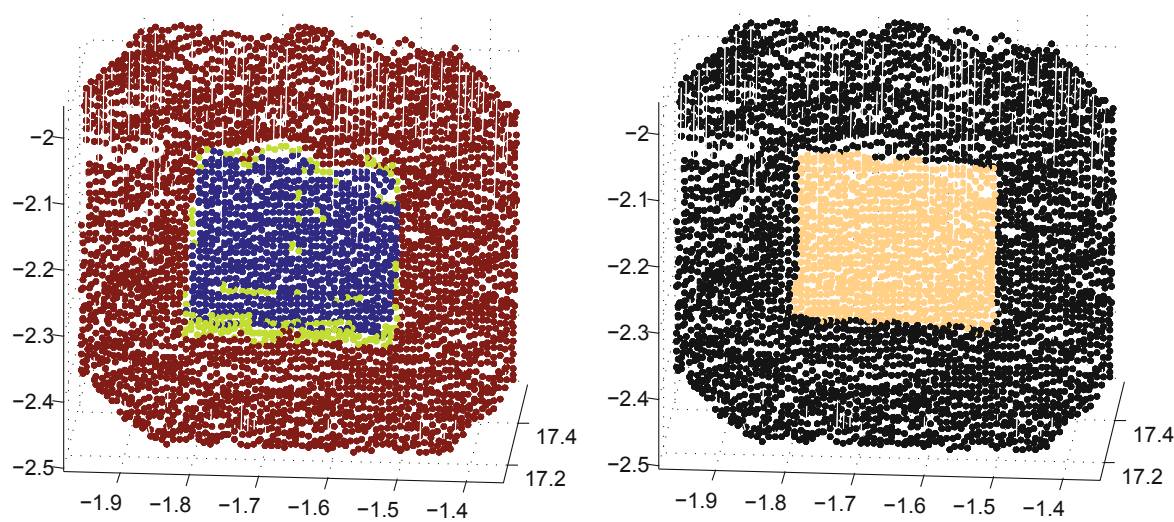

Fig. 2. Left: Segmentation result for the object shown in 1 consisting of three segments. Right: Ground truth.

to which each laser point belongs to a smooth surface patch. This measure is obtained through fitting of a local parabolic surface to each laser 3D sample and its neighbors. It is defined in terms of residual distance between the points and the surface and the normal direction similarity. For a particular sample $x, S_{x}$ is defined as

$$
S_{x}=\sum_{i \in \mathcal{N}_{x}}\left\langle\mathbf{n}_{x}, \mathbf{n}_{i}\right\rangle s_{i},
$$

where $\mathcal{N}_{x}$ defines the neighborhood of $x,\left\langle\hat{\mathbf{n}}_{x}, \mathbf{n}_{i}\right\rangle$ denotes the scalar product between the estimated normal at $x_{i}$ and the normal at a sample on the surface, and $s_{i}$ denotes the proximity between $x_{i}$ and the surface. $s_{i}$ equals 1 when the distance is zero and decreases for increasing distances and equals 0 beyond a distance threshold $\rho$. Hence, only points close to the surface and with normals similar to $\mathbf{n}_{x}$ contribute significantly to the surface score.

\subsection{Parameter Settings}

Throughout the examples, the following parameter settings were used. We set $k=5$ for all scenes. The final number of clusters, obtained after fusion of several features, was not limited. The threshold for removing low-weighted Gaussian functions was set to $\tau=0.01$. Assumptions of target dimensions resulted in 0.02 $\mathrm{m} \leq S C \leq 0.12 \mathrm{~m}$. The distance threshold for $S C$ was set to $\rho=0.04 \mathrm{~m}$, based on a priori knowledge of the range uncertainty of the laser scanner.

\section{Results}

The proposed method was applied on data from five scenes, where the mines were placed in forest, grass fields or on gravel road. There were a total of 126 targets of various sizes, from small anti-personnel mines (diameter 6-7 cm) to large anti-tank mines (about $30 \mathrm{~cm}$ ). The weighted average of the feature order for 


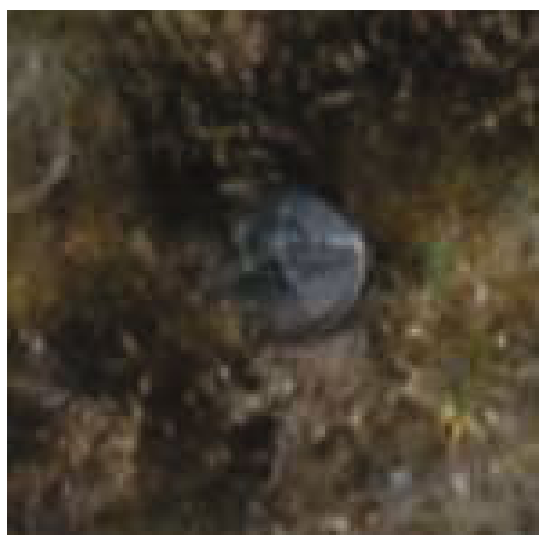

(a)

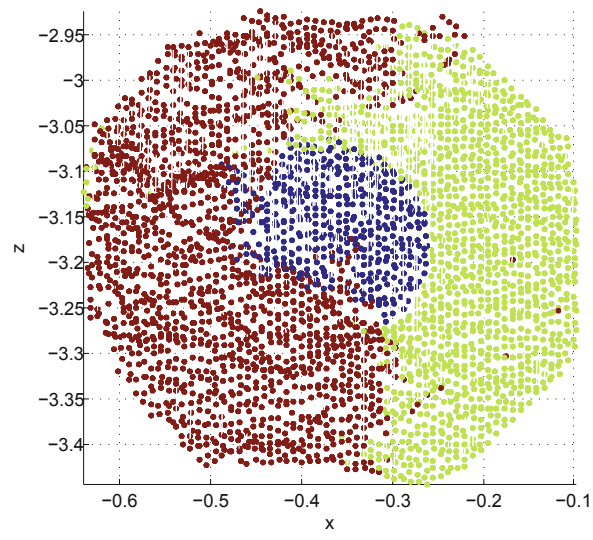

(c)

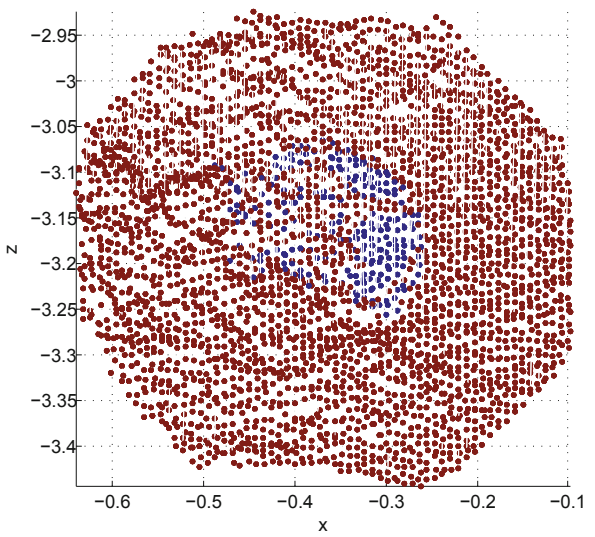

(b)

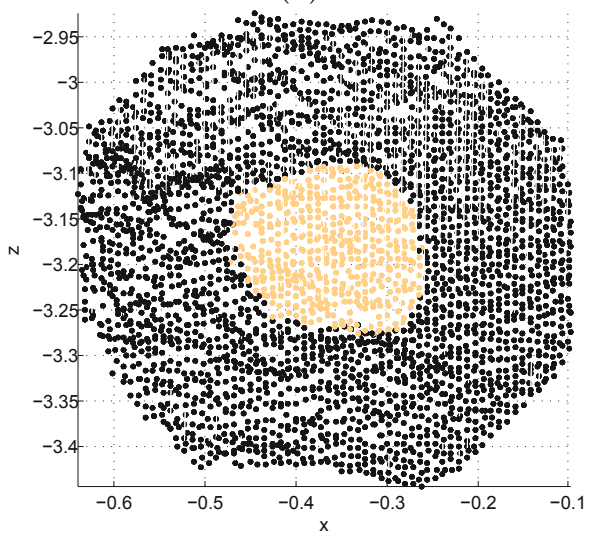

(d)

Fig. 3. (a) Image of a mine. (b) Segmentation result with the proposed method, based on the highest ranked feature (Intensity). (c) Final segmentation result with the proposed method (with four features: Intensity, Green, Height and Red). (d) Ground truth.

the five scenes is shown in Table 1. For scene five the RGB camera was not used and the MWIR data was preprocessed with the RX algorithm to one feature [6]. The feature order varies between the scenes, but features originating from laser scanner data get high ranking for all scenes. In the final segmentation result, all extracted segments of acceptable physical size were compared to ground truth data and the number of true target and non-target points, respectively, were counted. A total of 114 segments were found, of which 72 contained a majority of true target points. Detection statistics for scene 1 is shown in Fig. 4 , If there are more than 100 samples on the mine we get good performance. If there are less than 100 samples on the mine (mine 10-20), the segmentation sometimes fails in producing a good target-background segmentation. A closer inspection 
Table 1. Feature order (weighted average) for the five scenes. For scene 5 the RGB camera was not used and the MWIR data was preprocessed to one feature.

\begin{tabular}{|l|c|c|c|c|c|c|c|c|c|c|}
\hline \multirow{2}{*}{ Scene } & \multicolumn{10}{|c|}{ Features } \\
\cline { 2 - 14 } & Intensity & Height & Surface & Red & Green & Blue & MW1 & MW2 & MW3 & MW4 \\
\hline 1 (forest) & 1 & 2 & 5 & 6 & 4 & 3 & 10 & 7 & 9 & 8 \\
\hline 2 (forest) & 1 & 2 & 4 & 5 & 6 & 3 & 9 & 7 & 8 & 10 \\
\hline 3 (road) & 2 & 1 & 8 & 5 & 6 & 4 & 9 & 10 & 7 & 3 \\
\hline 4 (grass) & 1 & 3 & 6 & 5 & 4 & 2 & 7 & 8 & 10 & 9 \\
\hline 5 (forest) & 2 & 3 & 4 & - & - & - & 1 & 1 & 1 & 1 \\
\hline
\end{tabular}

of the result showed that of the remaining 42 segments, all but five corresponded to scenes containing small targets with only typically about $40-50$ samples. In other words, for these targets the segmentation was not able to find the target correctly. In addition to the targets, the algorithm was also applied on seven randomly chosen background areas (without any targets) and no acceptable clusters were found in any of them.
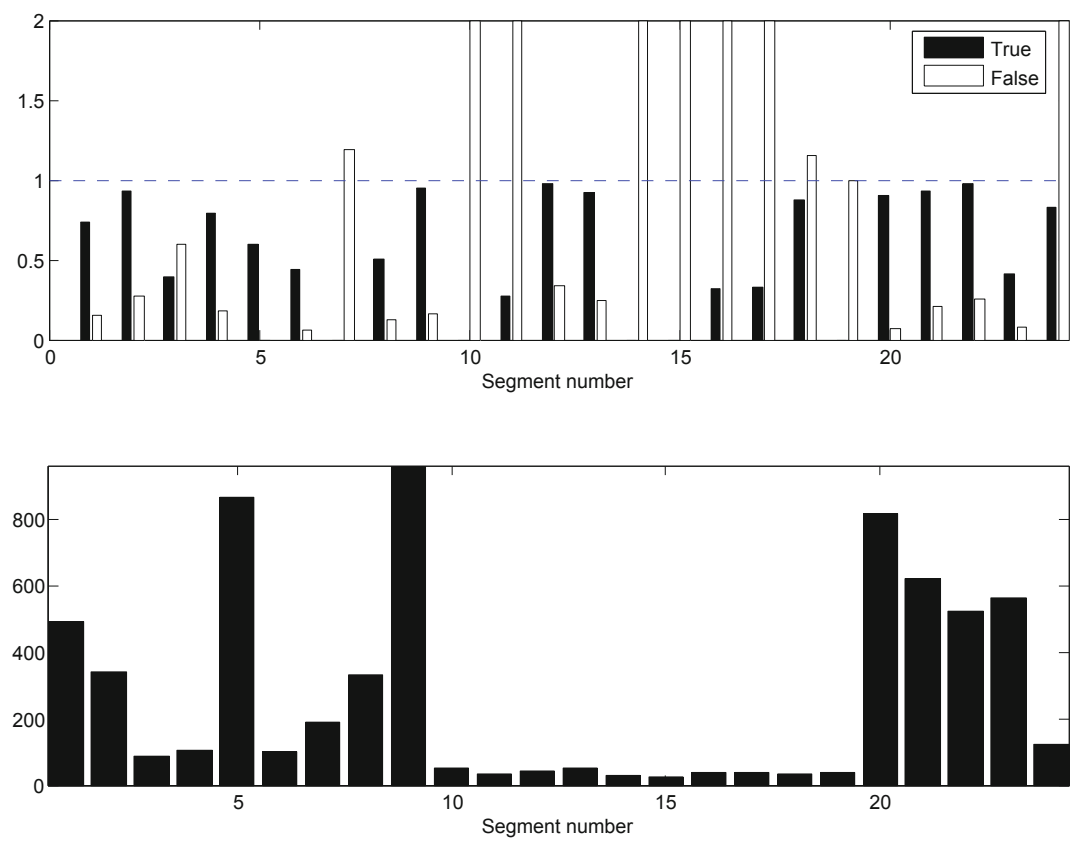

Fig. 4. Detection results for scene 1. Top: The number of true detections (black) and false detections (white) relative to the number of ground truth samples. For visualization purposes the scale has been truncated, and hence false detection values equal to two indicate that segments with at least twice the number of ground truth samples were found. Below: The number of ground truth samples for the targets in the scene. 


\section{Conclusions and Future Work}

In this paper, an approach to target-background segmentation based on fusion of optical sensor data is presented. The underlying source of the sensor data was assumed to be a mixture of Gaussians. The expectation maximization algorithm was used to estimate the parameters of the mixtures. Since the "true" number of mixtures is not known a priori, the minimum message length criterion was utilized to determine which of the Gaussian mixture estimations to use as a representation of the original data. Two criteria for quantifying clustering performance were investigated: a spatial scatter criterion and the normalized scatter separability criterion.

The method was tested on five scenes containing a total of 114 targets of which 72 were correctly segmented. The missed targets were mainly anti-personnel mines with a diameter smaller than $10 \mathrm{~cm}$. This implies that the segmentation method as such works quite well, but that there are data quality issues associated with small objects in cluttered environments. Not only are registration and ground truth errors more significant for smaller targets, but smaller objects tended to be more occluded than larger ones when placed in a cluttered environments (grass, sprigs, etc). Since the laser scanner has an inherent limitation to resolve multiple surfaces within the path of one laser pulse, noisy range readings are introduced for objects occluded by grass. Further, the range noise of the laser sensor is in the order of about $\sigma=1 \mathrm{~cm}$, which makes it difficult to accurately perform local spatial analysis for small objects, e.g. compute normal directions and the surface score measure.

Currently we use a fixed size of the neighborhood, chosen small to avoid smoothing effects for small objects, but a possible future improvement would be to perform a multi-scale spatial analysis or to adapt the size of the neighborhood according to the local statistics. Using a laser radar sensor with higher precision would most likely also improve the discrimitive power of the surface score.

It should be pointed out that the proposed method is a segmentation technique rather than a dedicated mine detector. In fact, the size of the segments (here measured in terms of a spatial scatter measure) is the only exploited target property. This means that as expected the method sometimes finds segments not corresponding to targets, but twigs, tufts of grass, etc. Including other properties of the targets, e.g. the fact that they are solid bodies, would most likely improve the results further. Further work will focus on how range data filtering approaches such as the ones presented in 14] could be incorporated in the process.

Currently, the clustering is performed in the feature space and the spatial distribution of points is used only for verification of the clustering results. A possible improvement would be to integrate the spatial relations between points into the clustering process itself. This could serve as a complement to the verification of cluster size that was proposed in this paper. 


\section{References}

1. Letalick, D., et al.: MOMS - Analysis and evaluation of experimental data, Tech. Rep., FOI, FOI-R-2012-SE (2006)

2. Letalick, D., et al.: MOMS - Progress report 2008, Tech. Rep., FOI, FOI-R-2622-SE (2008)

3. Oliver, J., Baxter, R., Wallace, C.: Unsupervised Learning Using MML. In: 13th International Conference on Machine Learning, pp. 364-372 (1996)

4. Dy, J.G., Brodley, C.E.: Feature Selection for Unsupervised Learning. Journal of Machine Learning Research 5, 845-889 (2004)

5. Figueiredo, M.A.T., Jain, A.K.: Unsupervised Learning of Finite Mixture Models. IEEE Trans. Pattern Analysis and Machine Intelligence 24(3), 381-396 (2002)

6. Tolt, G., Westberg, D., Grönwall, C.: A sensor fusion method for detection of surface laid mines. In: Swedish Symposium on Image Analysis, pp. 1-4 (2008)

7. Linderhed, A., Sjökvist, S., Nyberg, S., Uppsäll, M., Grönwall, C., Andersson, P., Letalick, D.: Temporal analysis for land mine detection. In: International Symposium on Image and Signal Processing and Analysis (ISPA), pp. 389-394 (2005)

8. Bali, N., Mohammad-Djafari, A.: Bayesian approach with hidden markov modeling and mean field approximation for hyperspectral data analysis. IEEE Trans. Image Processing 17, 217-225 (2008)

9. Jimenez, L.O., Landgrebe, D.A.: Hyperspectral data analysis and supervised feature reduction via projection pursuit. IEEE Trans. Geoscience and Remote Sensing 37, 2653-2667 (1999)

10. Constantinopoulos, C., Titsias, M.K., Likas, A.: Bayesian feature and model selection for Gaussian mixture models. IEEE Trans. Pattern Analysis and Machine Intelligence 28, 1013-1018 (2006)

11. Law, M.H.C., Figueiredo, M.A.T., Jain, A.K.: Simultaneous feature selection and clustering using mixture models. IEEE Trans. Pattern Analysis and Machine Intelligence 26, 1154-1166 (2004)

12. Mao, K.Z.: Identifying critical variables of principal components using unsupervised feature selection. IEEE Trans. Systems, Man and Cybernetics 35, 339-344 (2005)

13. Fauvel, M., Benediktsson, J.A., Chanussot, J., Sveinsson, J.R.: Spectral and Aptial classification of hyperspectral data using SVMs and morphological profiles. IEEE Trans. Geoscience and Remote Sensing 46, 3804-3814 (2008)

14. Grönwall, C., Tolt, G., Chevalier, T., Larsson, H.: Spatial filtering for detection of partly occluded targets. To be published in Optical Engineering, 50 (2011) 